\title{
Volatile organic compounds associated with Plasmodium falciparum infection in vitro
}

\author{
Ricardo Correa ${ }^{1,2}$, Lorena M. Coronado ${ }^{1,2}$, Anette C. Garrido 3 , Armando A. Durant-Archibold ${ }^{3}$ \\ and Carmenza Spadafora ${ }^{1 *}$ (I)
}

\begin{abstract}
Background: In order to identify new ways to prevent transmission of vector-borne diseases such as malaria, efforts have been made to understand how insects are attracted to humans. Vector-host interaction studies have shown that several volatile compounds play an important role in attracting mosquitoes to human targets. A headspace solid-phase micro-extraction/gas chromatography-mass spectrometry (HSPME GC-MS) analysis of the volatile organic composition of extracellular vesicles (EVs) and supernatants of ultracentrifugation (SNUs) was carried out in Plasmodium falciparum-infected cultures with high and low parasitemias.
\end{abstract}

Results: A list of 18 volatile organic compounds (VOCs) was obtained from the EVs of both infected and uninfected RBCs with 1,2,3-Propanetriol, diacetate (diacetin) increased in the infected EVs, regardless of the parasitemia of the culture. The supernatant analysis, however, gave off $56 \mathrm{VOCs}$, with pentane 2,2,4-trimethyl being present in all the SNUs of uninfected erythrocytes but absent from the parasite-infected ones. Standing out in this study was hexanal, a reported insect attractant, which was the only VOC present in all samples from SNUs from infected erythrocytes and absent from uninfected ones, suggesting that it originates during parasite infection.

Conclusions: The hexanal compound, reportedly a low-level component found in healthy human samples such as breath and plasma, had not been found in previous analyses of $P$. falciparum-infected patients or cultures. This compound has been reported as an Anopheles gambiae attractant in plants. While the compound could be produced during infection by the malaria parasite in human erythrocytes, the A. gambiae attraction could be used by the parasite as a strategy for transmission.

Keywords: Volatile organic compounds, HSPME, GC-MS, Plasmodium falciparum, Malaria, Vector

\section{Background}

Plasmodium falciparum is the protozoan parasite that causes the most severe variant of malaria cases worldwide [1]. The development of vector control strategies has been identified as an important pillar to decrease the malaria burden through transmission-reducing chemoprevention, correct insecticide applications and entomological surveillance [2]. Therefore, elucidating the mosquito's role in malaria transmission is a key factor to understanding the deadliest worldwide vector-borne disease. In addition, the

\footnotetext{
* Correspondence: cspadafora@indicasat.org.pa

${ }^{1}$ Center of Cellular and Molecular Biology of Diseases (CBCMe), Instituto de Investigaciones Científicas y Servicios de Alta Tecnología (INDICASAT AIP), City of Knowledge, Panama

Full list of author information is available at the end of the article
}

increase in drug-resistant parasites [3] and insecticideresistant mosquitoes [4] is driving scientists and policy makers to develop alternative mechanisms to reduce the transmission of $P$. falciparum.

There have been many efforts to decode how mosquitoes are attracted to humans in order to look for new ways to stop transmission. Vector-host interaction studies have shown that several chemical compounds play an important role in attracting the Anopheles spp. to human targets. These chemical attractants include $\mathrm{CO}_{2}[5]$, octenol [6], indole [7], ammonia [8], lactic acid and aliphatic carboxylic acids $[9,10]$, which are contained in human breath and sweat. The role of skin-associated 
microflora VOCs has also been identified as a potential source of vector attraction $[11,12]$.

However, there is evidence that additional signal attractants must play a role during malaria infection. New findings identified an augmented attraction of mosquitoes to malaria-infected patients, suggesting a possible parasite manipulation on the physicochemical activity of the host [13-15] but this influence on humans by $P$. falciparum is not yet well understood.

Recent studies have been conducted to compare the volatile chemical burden during the asexual stage of $P$. falciparum in in vitro cultures. One study did not find any difference between $P$. falciparum-infected and noninfected RBCs [16]. However, a more recent report revealed the presence of several $P$. falciparum-associated terpenes. Notably, one of them, pinene, was found in very low quantities in infected red blood cells (iRBCs) [17]. Interestingly, pinene was previously reported as an A. gambiae attractant present in plants during sugar feeding [18]. Nevertheless, further analyses are needed to correlate that finding with the possibility of a host manipulation by the parasite to produce vector attraction signals during malaria infection.

The taking over of the host cell machinery by pathogens has been reported during several intracellular infections in bacteria, mammals and plants [19-22]. One such special control mechanism is the release of extracellular vesicles (EVs) to improve survival of the pathogen, as in macrophage infection by Leishmania parasites [23, 24]. The extracellular vesicles released from iRBCs during malaria infection have also been studied over the last few years, revealing that EVs are capable of transporting Plasmodium spp. molecules (RNA, DNA, protein and lipids) [25-29]. However, the VOC load in EVs has not been characterized in any malaria report thus far. Therefore, this study aimed to identify a possible differential content of EVs-VOCs in in vitro cultures of $P$. falciparum growing at two different parasitemias, and the probable relationship between the higher EV-VOC density and mosquito attraction.

\section{Methods}

We cultured the P. falciparum HB3 strain using the conventional method of Trager \& Jensen [30] with modifications described in Almanza et al. [31], that include the use of modified RPMI 1640 medium (Sigma-Aldrich, St. Louis, USA), $25 \mathrm{mM}$ HEPES, $15 \mu \mathrm{M}$ hipoxanthine, $50 \mathrm{mg} / \mathrm{ml}$ gentamicine sulfate, and $200 \mathrm{mM}$ L-Glutamine, supplemented with $10 \%$ human serum, $2 \%$ sodium bicarbonate and a mix of gases $\left(90 \% \mathrm{~N}_{2}, 5 \% \mathrm{O}_{2}\right.$ and $\left.5 \% \mathrm{CO}_{2}\right)$. Synchronization was performed in a temperature-cycling incubator (TCI) (Cooled Incubator, Sanyo, Model MIR154 ) and by the addition of $0.3 \mathrm{M}$ alanine (Sigma-Aldrich). Uninfected red blood cells (uRBCs) were cultured with the same hematocrit (2\%) and media conditions.
Microvesicles were obtained from $25 \mathrm{ml}$ of infected and uninfected RBCs from three volunteers. Supplementation media for each T75 culture bottle used the corresponding serum from each volunteer. Two replicas of $\mathrm{uRBCs}$ and of low ( 4\%) and high (15-30\%) iRBC parasitemia were prepared from the blood of each volunteer. Parasitemia was evaluated by optical microscopy using Giemsa staining (GS500, Sigma-Aldrich). The procedure for isolation of microvesicles was based on a parasitic EV isolation report [32]. The iRBC and uRBC cultures were collected and centrifuged at $2000 \times g$ for $15 \mathrm{~min}$. The $2000 \times g$ supernatants were then centrifuged at $15,000 \times g$ at $4{ }^{\circ} \mathrm{C}$ for $30 \mathrm{~min}$ to remove cell debris. Next, these supernatants were filtered through $0.2 \mu \mathrm{m}$ low-binding protein filters (Acrodisc, Pall Life Science, Port Washington, USA) and the filtered-supernatants were ultracentrifuged at 110 , $000 \times g 4{ }^{\circ} \mathrm{C}$ for $70 \mathrm{~min}$ to pellet small vesicles. The pellet was washed once by resuspending it in sterile doublefiltered $(0.2 \mu \mathrm{m})$ PBS $1 \mathrm{X}$ and further ultracentrifuged at $110,000 \times g$ for an additional $70 \mathrm{~min}$. The pellet was resuspended in $100 \mu \mathrm{l}$ of double-filtered PBS $1 \times$ for analysis, discarding the supernatant. At the same time, $20 \mathrm{ml}$ of the supernatant formed in the first ultracentrifugation (SNU) were collected and concentrated to $\sim 500 \mu \mathrm{l}$ at $3000 \times g$ for $2 \mathrm{~h}$ using $3 \mathrm{kDa}$ Vivaspin tubes.

The size-characterization of EVs by flow cytometry (CyFlow, Partec, Kent, UK) was performed using a similar procedure and parameters to those that have been used to measure microvesicles in plasma [33]. The EVs, which were characterized by measuring forward scatter (FSC) size and side scatter (SSC) granularity, were added immediately prior to analysis by flow cytometry. We established gates based on region size by calibrating the gain of polyethylene beads of different sizes $(0.1,0.5$ and $2 \mu \mathrm{m})$ (Fluka Analytical, Sigma-Aldrich) before measuring the samples. We determined the background noise per second with $750 \mu \mathrm{l}$ of double-filtered $(0.2 \mu \mathrm{m})$ phosphate-buffered saline (PBS) solution. Data were acquired and analyzed using FloMax software. The final concentration of the samples was calculated using the software's True Volumetric Absolute Counting system, based directly on the basic definition of concentration $\mathrm{c}=\mathrm{N} / \mathrm{V}$, using an electrode-principle determination.

Independent cultures at high and low parasitemia were collected to perform western blot analysis in order to detect the presence of CD63 (System Biosciences, SBI, Exo AB kit-1, Palo Alto, USA), a conserved extracellular vesicle protein commonly used as an EV marker, and PfMSP1 (Abcam, ab156840, Cambridge, UK) which is a merozoite membrane protein like the AMA1 used by Mantel et al. [29]. Also, Glycophorin A,B ( \#G7650, SigmaAldrich), the main glycoprotein in the red blood cell membrane, was used. The antibodies were diluted as instructed by each manufacturer. 
Samples were analyzed by HSPME-GC-MS. A $1.5 \mathrm{ml}$ vial containing the sample was sealed and the SPME extraction was performed with a DVD/CAR/PDMS fiber (Supelco, Bellefonte, PA, USA). All HSPME extractions were done at $37{ }^{\circ} \mathrm{C}$ for $12 \mathrm{~h}$. All samples were analyzed by gas chromatography-mass spectrometry analyses (GC-MS), using an Agilent $6890 \mathrm{~N}$ gas chromatographer connected to a 5975C triple-axis mass selective detector (Agilent Technologies, Palo Alto, CA, USA). Samples were injected in splitless mode, with the injector temperature set at $250{ }^{\circ} \mathrm{C}$. The GC column used for the study was an HP-5MS, $30 \mathrm{~m}$ length, $0.25 \mathrm{~mm}$ i.d. and $0.25 \mu \mathrm{m}$ phase thickness (Agilent Technologies, Palo Alto, CA, USA). Helium was used as carrier gas at $1.0 \mathrm{ml} / \mathrm{min}$. The temperature gradient began with an initial temperature of $50{ }^{\circ} \mathrm{C}$, held for $3 \mathrm{~min}$, increased to $200{ }^{\circ} \mathrm{C}$ at $6{ }^{\circ} \mathrm{C} \mathrm{min}{ }^{-1}$, and finally upped to $280{ }^{\circ} \mathrm{C}$ at $10^{\circ} \mathrm{C} \mathrm{m^{-1 }}$. Mass spectrometry detection was performed in the EI mode, with the ion source temperature, electron energy, and transfer line temperature set at $250{ }^{\circ} \mathrm{C}, 70 \mathrm{eV}$, and $280{ }^{\circ} \mathrm{C}$, respectively. Identification of all compounds was done based on their fragmentation patterns using authentic standards when available, and the NIST 11 data base using mass spectral deconvolution and identification system (AMDIS). For further identification, the retention indices of each compound were compared with those reported in the literature.

\section{Results}

The criteria used to choose the VOCs to which an abundance determination was applied was based on their presence in at least one of the technical replicates of all three volunteers. The comparison between their uRBCs and iRBCs levels was based on the detection of VOCs in the analysis of all samples [technical replicates of each parasitemia ( 2 high and 2 low) and 2 of uRBCs; all from 3 biological samples] from collected EVs, and their respective SNUs. In light of the limited amount of EVs collected from uRBCs, the two technical replicates of EVs from each volunteer were pooled before analyzing this population. This resulted in a total of $15 \mathrm{EV}$ samples (3 uRBCs and 12 iRBCs) and 18 SNUs.

The concentration of EVs varied from sample to sample (see Table 1 and Additional file 1: Table S1). Around 98\% of the EVs had an average size of $0.1 \mu \mathrm{m}$ when they were compared with standard microbeads. A reduced

Table 1 Concentration and size distribution of EV samples

\begin{tabular}{|c|c|c|c|}
\hline & uRBCs & $\begin{array}{l}\text { iRBCs Low } \\
\text { Parasitemia }\end{array}$ & $\begin{array}{l}\text { iRBCs High } \\
\text { Parasitemia }\end{array}$ \\
\hline Size & $\mathrm{EV} / \mathrm{ml}$ (SD) & EV/ml (SD) & $\mathrm{EV} / \mu \mathrm{l}(\mathrm{SD})$ \\
\hline $0.1 \mu \mathrm{m}$ & $74,331(13,418.9)$ & $77,970(19,424.8)$ & $200,232(61,325.4)$ \\
\hline $0.5 \mu \mathrm{m}$ & 927 (812) & 1094 (373.1) & $1848(1557.3)$ \\
\hline $2.0 \mu \mathrm{m}$ & 395 (295.5) & 755 (362.2) & $1326(1053.7)$ \\
\hline
\end{tabular}

number (2\%) of EVs fell in either the $0.5 \mu \mathrm{m}$ or the $2 \mu \mathrm{m}$ regions. In addition, the identity of the EVs after isolation was confirmed through detection of the human extracellular vesicle conserved marker CD63 and the parasite-specific membrane protein PfMSP1 by Western blot (Fig. 1). The typical bands below $30 \mathrm{kDa}$ (one in duplet) [34], the $43 \mathrm{kDa}$ [35] and a band above $50 \mathrm{kDa}$, as shown by the manufacturer, are all present in the blot. The MSP1 Western blot also reveals the expected $19 \mathrm{kDa}$ band [36] (and in manufacturer's site) only in lysates or EVs from infected cultures. In addition, Glycophorin A (GPA), a protein abundant in the membrane of red blood cells, was used to confirm the collected vesicles' erythrocytic origin. A number of bands usually due to various glycosilation and dimerization states showed up in the blot [37], but most importantly, the EVs collected in this study were also rich in GPA and confirmed the source of the samples. The established protocol used to collect the EVs, their size determination by flow cytometry and the presence of GPA, PfMSP1 and CD63 in the population, altogether, confirm that our samples are extracellular vesicles from the parasite-infected erythrocytes.

The identity of the VOCs found in this study was characterized after comparing the spectral fragmentation patterns with standards and subjecting the results to spectral deconvolution. Then, the retention indices, which are maintained across different types of columns, equipments and settings, were compared to those in the NIST 11 database (Table 2). In the analysis of EVs from iRBCs and uRBCs (Additional file 2: Table S2), one of the most abundant VOCs was 2-ethyl-1-Hexanol (Table 3), which is associated with plastic contamination [16]. Another major compound in our analysis was 1,3-bis(1,1-dimethylethyl)Benzene, likely produced after gamma irradiation is applied to powder supplements in commercial RPMI [38]. There were no VOCs exclusive to EVs derived from iRBCs after analyzing all biological and technical replicas, although several molecules were present exclusively in one or more technical replicas of the iRBCs from a single volunteer (Additional file 2: Table S2).

Interestingly, 1,2,3-Propanetriol diacetate (Diacetin) was a commonly present compound on EVs from infected cultures, having at least 9 hits, reaching an average of $17.8 \%$ of the total parasitic EV VOCs according to the total area sum (Table 3). Notably, while Diacetin was found in most samples from infected cultures, it also appeared in one technical replica of an uninfected volunteer. Other VOCs discovered only on the iRBCs were benzeneacetaldehyde, butanoic acid, butyl ester, ethylbenzene and o-xylene, however they only showed up once in our analysis, while several other compounds in the iRBCs, as shown in our databases, also showed up in most SNUs or EVs from uRBCs, as the alkenes undecane, tridecane, tetradecane and dodecane (Additional file 2: Table S2; Additional file 3: Table S3). 

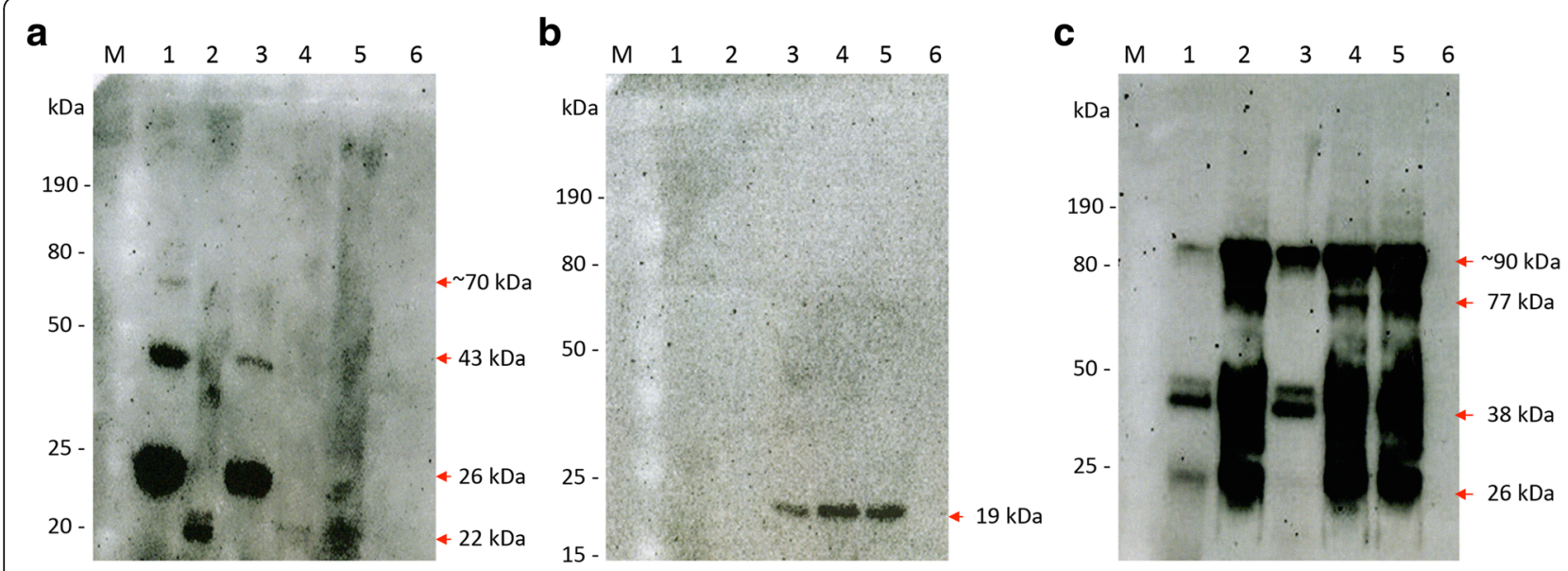

Fig. 1 Extracellular vesicles (EVs) are recognized by common markers. Erythrocytes and EVs were lysed with RIPA buffer. Each lane was loaded with $20 \mathrm{\mu g}$ of protein sample, as assessed by a Bradford assay, subjected to non-denatured (a) or SDS PAGE (b, c), transferred to polyvinyl membranes and probed with antibodies against CD63 (a), PfMSP1 (b) and Glycophorin A (c). CD63 specific primary antibodies were used at 1:1000 dilution and a secondary Goat anti-Rabbit IgG HRP conjugated antibody (System Bioscience) was used at 1:20,000 dilution. MSP1 was used at 1:50 dilution with a secondary Mouse lgG HRP-conjugated antibody (R\&D System) at a 1:1000 dilution. To detect Glycophorin, the E3 clone was used following the manufacturer instructions. The gel lanes were loaded as follows: M: size marker; Lane 1: uninfected erythrocytes lysate; Lane 2: EVs from uninfected erythrocytes; Lane 3: infected erythrocytes lysate; Lane 4: EVs from infected erythrocytes at low parasitemia; Lane 5: EVs from infected erythrocytes at high parasitemia; Lane 6: Human serum. Red arrows mark the bands corresponding to the bands expected

The analysis of SNUs was originally performed to distinguish VOCs in supernatants from those associated to the EVs. However, a higher number of VOCs was found in SNUs. We identified 56 different VOCs with variable frequencies (Additional file 3: Table S3). Only 17 compounds fulfilled the criteria for the analysis in this study

Table 2 Experimental and theoretical retention indices of the VOCs

\begin{tabular}{lll}
\hline VOCs & Theoretical IR & Experimental IR \\
\hline 2,2,4-trimethyl-pentane & 691 & 700 \\
Hexanal & 801 & 809 \\
1-Octen-3-ol & 979 & 987 \\
Nonanal & 1104 & 1111 \\
2-ethyl-1-hexanol & 1020 & 1028 \\
Isoborneol & 1160 & 1167 \\
Menthol & 1171 & 1179 \\
a-Terpineol & 1192 & 1200 \\
Dodecane & 1200 & 1208 \\
1,2,3-propanetriol, diacetate & 1230 & 1236 \\
1,3-bis(1,1-dimethylethyl)-benzene & 1249 & 1258 \\
p-tert-butyl-phenol & 1256 & 1262 \\
Isobornyl acetate & 1288 & 1296 \\
Bornyl acetate & 1290 & 1296 \\
Tridecane & 1300 & 1309 \\
Tetradecane & 1399 & 1407 \\
B-lonone & 1489 & 1498 \\
Butylated hydroxytoluene & 1516 & 1523 \\
\hline
\end{tabular}

(Table 4). The presence of the same contaminants found in the EV samples was also detected in SNUs. Of all the samples analyzed, hexanal appeared to be associated almost exclusively with SNUs from the iRBCs of all three volunteers, being absent in all replicas of uRBCs. Conversely, 2,2,4-trimethyl-pentane was found in all of the SNUs from the uRBCs but was completely absent from those of the iRBCs. Several terpenes such as isoborneol, borneol and menthol were often found in parasitized samples, possibly originating from the types of foods eaten by the volunteers prior to drawing their blood. These terpenes show a high variability among their relative areas, representing anywhere from 0.01 to $41.5 \%$ of them in any one sample. Notably, 1-octen-3-ol (a known mosquito attractant) was found to be increased in SNUs from iRBCs, although there was a high variability among samples.

Table 3 Abundance of VOCs of EVs (uRBCs and iRBCs). The total sum of all the peak areas of each compound in the chromatograms of all assays is listed. Hits are the number of replicates in which the compound was found

\begin{tabular}{lll}
\hline VOCs & $\begin{array}{l}\sum \text { Area of uRBCs } \\
\text { (hits out of 3 } \\
\text { maximum } \\
\text { replicates) }\end{array}$ & $\begin{array}{l}\sum \text { Area of iRBCs } \\
\text { (hits out of 12 } \\
\text { maximum } \\
\text { replicates) }\end{array}$ \\
\hline 2-ethyl-1-Hexanol & $3,737,076(3)$ & $17,333,851(12)$ \\
1,3-bis(1,1-dimethylethyl)- Benzene & $3,737,076(3)$ & $13,753,288(9)$ \\
1,2,3-Propanetriol, diacetate & $1,509,468(1)$ & $12,786,280(9)$ \\
Dodecane & $967,212(1)$ & $5,347,592(3)$ \\
\hline
\end{tabular}


Table 4 Abundance of VOCs from SNUs (URBCs and iRBCs). The total sum of all the peak areas of each compound in the chromatograms of all assays is listed. Hits are the number of replicates in which the compound was found

\begin{tabular}{lll}
\hline VOCs & $\sum$ Area of uRBCs (hits out of 6 maximum replicates) & $\sum$ Area of iRBCs (hits out of 12 maximum replicates) \\
\hline 1,3-bis(1,1-dimethylethyl)-Benzene & $175,199,553.0(6 / 6)$ & $280,310,131.0(12 / 12)$ \\
Isoborneol & $175,199,553.0(6 / 6)$ & $240,190,385.0(11 / 12)$ \\
2-ethyl-1-Hexanol & $175,199,553.0(6 / 6)$ & $280,310,131.0(12 / 12)$ \\
Dodecane & $175,199,553.0(6 / 6)$ & $280,310,131.0(12 / 12)$ \\
Tetradecane & $175,199,553.0(6 / 6)$ & $280,310,131.0(12 / 12)$ \\
p-tert-butyl-Phenol & $175199553.0(6 / 6)$ & $280,310,131.0(12 / 12)$ \\
a-Terpineol & $175,199,553.0(6 / 6)$ & $280,310,131.0(12 / 12)$ \\
1-Octen-3-ol & $140,850,914.0(4 / 6)$ & $280,310,131.0(12 / 12)$ \\
Nonanal & $175,199,553.0(6 / 6)$ & $212,397,351.0(8 / 12)$ \\
Menthol & $161,415,286.0(5 / 6)$ & $243,569,806.0(11 / 12)$ \\
Isobornyl acetate & $146,838,216.0(4 / 6)$ & $269,475,284.0(11 / 12)$ \\
Hexanal & $0 / 6$ & $280,310,131.0(12 / 12)$ \\
trans- $\beta$-lonone & $28,162,111.0(1 / 6)$ & $165,488,621.0(7 / 12)$ \\
Tridecane & $86,806,175.0(1 / 6)$ & $86,803,949.0(3 / 12)$ \\
Bornyl acetate & $28,361,337.0(2 / 6)$ & $10,834,847.0(1 / 12)$ \\
2,2,4-trimethyl-pentane & $175,199,553.0(6 / 6)$ & $(0 / 12)$ \\
Butylated hydroxytoluene & $(0 / 6)$ & $86,803,949.0(3 / 12)$ \\
\hline
\end{tabular}

A graph of the three main findings of this study is presented in Fig. 2 where the difference in expression or production of each VOC is stressed.

\section{Discussion}

The hypothesis of host manipulation by the parasite to induce mosquito-attractant VOCs through EVs was tested due to the capacity of the latter to transport key signal molecules. In this study, EVs isolated from P. falciparum- infected cultures, and the supernatants of those cultures at two different parasitemias, were analyzed for their VOC content. To validate the source of the compounds, immunoblots were used with common markers of extracellular vesicles [39]. The blots revealed that CD63 is enriched in all EVs, regardless of infection by the pathogen. The merozoite membrane protein MSP1, cleaved by serine proteases to give its $19 \mathrm{kDa}$ size [36], is present only in extracellular vesicles from iRBCs, as expected, and is also
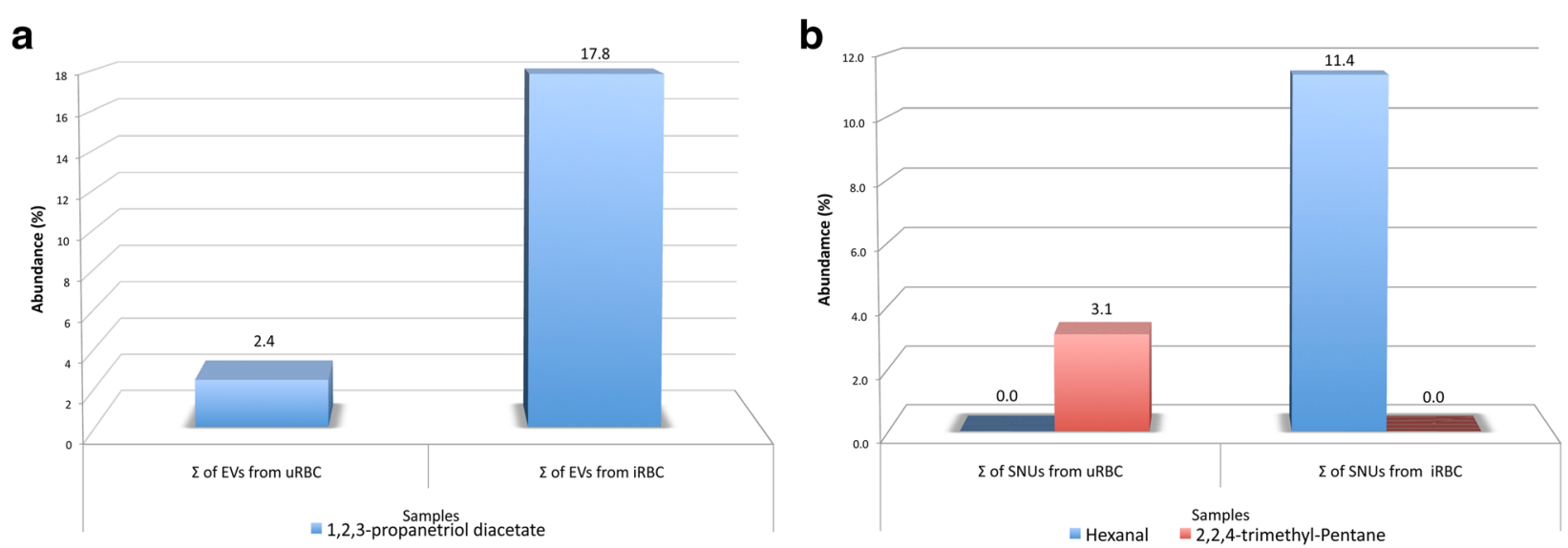

Fig. 2 Difference in abundance of VOCs in P. falciparum-infected vs uninfected cultures. The peak areas of three volatile organic compounds, a 1,2,3-propanetriol diacetate (found in the EVs) from URBC and iRBC and $\mathbf{b}$ 2,2,4-trimethyl-pentane and hexanal (found in SNUs) from URBC and iRBC were summed up across all replicates and number of experiments. The total area thus found was compared between uninfected samples and infected ones 
present in the lysate of infected cultures. We have added a third marker to detect our extracellular vesicles: Glycophorin A. GPA is one of the most abundant proteins in the membrane of erythrocytic cells, and has numerous Nand O-glycosylation sites and also several polymorphic and quaternary structural presentations, making it migrate at different speeds on the polyacrylamide gels, depending on its state. We detected the presence of GPA in the cell lysates and in the EVs of all samples of red blood cell origin, demonstrating the usefulness of this marker as well for erythrocytic EVs.

As for the analysis of volatile material, a different methodology from those previously used to analyze standard or larger volumes of culture $[16,17]$ was used, which uncovered compounds not previously described as associated with P. falciparum or with its EVs. In addition, the use of human sera to culture the parasite offered the opportunity to perform VOC analysis in a more realistic environment in comparison with Albumax supplementation used in previous studies. In this scenario, the biological replicas of three human volunteers allowed us to understand conserved or non-conserved VOC production during infection by $P$. falciparum. The main obstacle faced in the study was the high concentration of possible contaminant compounds that could mask other less abundant VOCs. Most of these contaminants were present in both samples, suggesting an extrinsic origin derived from the culture media or plastic containers.

The high diversity of peak areas displayed by the replicas for each detected VOC implies a greater complexity than originally anticipated, which is independent of the level of parasitemia. The presence of diacetin in the vast majority of the EVs from iRBCs suggests a possible function in malaria infection. It is tempting to propose a role for this compound in the attraction of insects, given the recent report of diacetin being a phyto-attractant of oil bees [40], although more evidence is needed to verify this supposition. Additional compounds that were exclusive of the EVs from iRBCs did not have enough reproducibility to stand out given that they showed up in the iRBCs of only one out of the three volunteers.

The main difference in VOCs from uRBCs and iRBCs cultures was found in SNUs, even though the SNUs were initially intended to be used only as controls to differentiate the VOC-EV association. The terpenes found in our SNU samples were different from those reported to have mosquito attraction capacity, such as pinene [17]. Arguably, pinene and other terpenes not present in our samples - which contained only EVs or SNUs could have their origin in the erythrocytes present in the sample when Kelly et al. [17] conducted their VOC search. This would explain why pinene, for example, has been found in blood from healthy volunteers [41].
Interestingly, the known solvent 2,2,4-trimethyl-pentane, present probably as a contaminant in the samples of uninfected RBCs, disappeared from the infected ones, as if the presence of the parasites had an influence on the decay of the compound.

As for the SNUs, hexanal was present in all of the samples of infected RBCs from both high and low parasitemia cultures. This compound was recently identified as a strong phyto-attractant of A. gambiae [18] and has been tested in mosquito baits [42]. Hexanal has been reported in several studies as a common marker in human breath and skin emanations, though these studies did not find it in the blood of healthy volunteers [41, 43-45]. Other technical approaches to the VOC content in humans, however, did find hexanal in normal plasma and breath, although at very low concentrations in comparison with the increased levels of hexanal connected to lung cancer patients [46-48], apparently linking its presence to cellular dysfunction or injury. Intriguingly, a recent study in humans infected with $P$. falciparum did not report hexanal in the breath of infected volunteers [49]. Although this VOC has been detected in in vitro cultures of Plasmodium vinckei [50], there have been as yet no reports on the generation of hexanal during $P$. falciparum infection. This compound is likely formed during membrane lipid peroxidation of cells as reported by Keller et al. [51], which would include RBCs in stress conditions [52] such as those encountered during parasitic infection. With its demonstrated attractiveness to the Anopheles mosquito, the production of hexanal could be advantageous to parasite transmission capabilities although studies are needed to test hexanal production in vivo in order to determine whether it plays a significant role in attracting mosquitoes to malaria-infected patients.

\section{Conclusions}

An HSPME GC-MS analysis of extracellular vesicles and supernatants of $P$. falciparum-infected and uninfected RBCs was conducted. Close to 100 volatile organic compounds were detected which varied in their proportion and presence in the samples. Notably, diacetin, an insect attractant found in plants, was present in most of the extracellular vesicles of infected RBCs but found only once in the EVs of a healthy RBC sample, suggesting a possible role during malaria infection. Additionally, hexanal, not found in previous analyses of P. falciparum-infected patients, was present in our study in all supernatants coming from infected blood but absent in those from uninfected blood. It is noteworthy that hexanal has been described as an A. gambiae attractant in plants and, although it is possible that this compound is a by-product of the process of 
parasitic infection of the erythrocytes, its mosquitoattraction capabilities could be used by the parasite as a strategy to increase the likelihood of transmission. Although more studies are required in patients infected with $P$. falciparum to confirm these findings, this information could be significant in the development of strategies aimed at preventing transmission by offsetting the parasite's vector-attracting capabilities.

\section{Additional files}

Additional file 1: Table S1. Characterization by flow cytometry. Raw readings show the size of isolated extracellular vesicles (EVs from three volunteers (biological replicates). In addition, PBS 1X was also analyzed to eliminate the buffer background. Numbers were obtained after averaging the raw data. (XLSX $50 \mathrm{~kb}$ )

Additional file 2: Table S2. Detection of VOCs in extracellular vesicles (EVs). Raw data show the abundance area of each VOC in every sample from all volunteers. The total sample area is the sum of all samples. (XLSX $14 \mathrm{~kb})$

Additional file 3: Table S3. Detection of VOCs in SNUs. Raw data show the abundance area of each VOC in each sample from all volunteers. The total area sample was summed up for each sample. (XLSX 20 kb)

\section{Abbreviations}

EV: Extracellular vesicle; HSPME GC-MS: Headspace solid-phase microextraction/gas chromatography-mass spectrometry; iRBC: Infected red blood cells; RBC: Red blood cells; SNU: Supernatant of ultracentrifugation; URBC: Uninfected red blood cells; VOC: Volatile organic compound

\section{Acknowledgements}

The authors would like to acknowledge the contribution of Rebecca Wentzel in proof-reading the manuscript.

\section{Funding}

These project was supported by a research grant by the International Centre of Genetic Engineering and Biotechnology (www.icgeb.org) (CRP/ 14 / 013) to CS; the Secretaría Nacional de Ciencia, Tecnología e Innovación (www.senacyt.gob.pa) together with the Instituto para la Formación y Aprovechamiento de los Recursos Humanos-Panamá Doctoral scholarship to LMC and RC; and the National System of Investigators of Panama grants 33-2015 and 170-2016 to AADA and CS, respectively.

\section{Availability of data and materials}

All the data collected in this study are available in the article and its Additional files.

\section{Authors' contributions}

Conceived and designed the experiments: RC, AADA and CS. Performed the experiments: RC, LMC and ACG. Analyzed the data: RC. Wrote the paper: RC, AADA and CS. All authors read and approved the final manuscript.

\section{Competing interests}

The authors declare that they have no competing interests.

\section{Consent for publication}

Not applicable

\section{Ethics approval and consent to participate}

Blood was donated at a point of care facility from healthy volunteers after signing a written consent.

\section{Publisher's Note}

Springer Nature remains neutral with regard to jurisdictional claims in published maps and institutional affiliations.

\section{Author details}

'Center of Cellular and Molecular Biology of Diseases (CBCMe), Instituto de Investigaciones Científicas y Servicios de Alta Tecnología (INDICASAT AIP), City of Knowledge, Panama. ${ }^{2}$ Department of Biotechnology, Acharya Nagarjuna University, Guntur 522 510, AP, India. ${ }^{3}$ Molecular Medicine Research Unit, Center for Biodiversity and Drug Discovery, Instituto de Investigaciones Cientificas y Servicios de Alta Tecnologia (INDICASAT AIP), City of Knowledge, Panama.

Received: 11 December 2016 Accepted: 25 April 2017

Published online: 02 May 2017

\section{References}

1. World Health Organization. World Malaria Report (2008-2016). Geneva: World Health Organization; 2016. [http://www.who.int/malaria/publications/ world_malaria_report/en/]. Accessed 20 May 2017.

2. World Health Organization. Global Technical Strategy for Malaria 2016-2030. Geneva: World Health Organization; 2015. http://www.who.int/malaria/ areas/global_technical_strategy/en/]. Accessed 20 May 2017.

3. Wongsrichanalai $\mathrm{C}$, Sibley $\mathrm{CH}$. Fighting drug-resistant Plasmodium falciparum: the challenge of artemisinin resistance. Clin Microbiol Infect. 2013;19(10):908-16.

4. Constant VAE, Benjamin GK, Christopher MJ, David W, Hilary R. Multipleinsecticide resistance in Anopheles gambiae mosquitoes, Southern Côte d'Ivoire. Emerg Infect Dis. 2012;18(9):1508.

5. Webster B, Lacey ES, Carde RT. Waiting with bated breath: opportunistic orientation to human odor in the malaria mosquito, Anopheles gambiae, is modulated by minute changes in carbon dioxide concentration. J Chem Ecol. 2015;41(1):59-66.

6. Takken W, Kline DL. Carbon dioxide and 1-octen-3-ol as mosquito attractants. J Am Mosq Control Assoc. 1989;5(3):311-6.

7. Meijerink J, Braks MAH, Brack AA, Adam W, Dekker T, Posthumus MA, et al. Identification of olfactory stimulants for Anopheles gambiae from human sweat samples. J Chem Ecol. 2000;26(6):1367-82.

8. Meijerink J, Braks MAH, Van Loon JJA. Olfactory receptors on the antennae of the malaria mosquito Anopheles gambiae are sensitive to ammonia and other sweat-borne components. J Insect Physiol. 2001;47(4-5):455-64.

9. Smallegange RC, Qiu YT, van Loon JJ, Takken W. Synergism between ammonia, lactic acid and carboxylic acids as kairomones in the host-seeking behaviour of the malaria mosquito Anopheles gambiae sensu stricto (Diptera: Culicidae). Chem Senses. 2005;30(2):145-52.

10. Smallegange RC, Qiu YT, Bukovinszkine-Kiss G, Van Loon JJ, Takken W. The effect of aliphatic carboxylic acids on olfaction-based host-seeking of the malaria mosquito Anopheles gambiae sensu stricto. J Chem Ecol. 2009;35(8):933-43.

11. Verhulst NO, Beijleveld H, Knols BG, Takken W, Schraa G, Bouwmeester HJ, et al. Cultured skin microbiota attracts malaria mosquitoes. Malar J. 2009:8:302.

12. Braks MA, Anderson RA, Knols BG. Infochemicals in mosquito host selection: human skin microflora and Plasmodium parasites. Parasitol Today. 1999; 15(10):409-13.

13. De Moraes CM, Stanczyk NM, Betz HS, Pulido H, Sim DG, Read AF, et al. Malaria-induced changes in host odors enhance mosquito attraction. Proc Natl Acad Sci USA. 2014;111(30):11079-84.

14. Lacroix R, Mukabana WR, Gouagna LC, Koella JC. Malaria infection increases attractiveness of humans to mosquitoes. PLoS Biol. 2005;3(9):e298.

15. Cornet S, Nicot A, Rivero A, Gandon S. Malaria infection increases bird attractiveness to uninfected mosquitoes. Ecol Lett. 2013;16(3):323-9.

16. Wong RP, Flematti GR, Davis TM. Investigation of volatile organic biomarkers derived from Plasmodium falciparum in vitro. Malar J. 2012;11:314.

17. Kelly M, Su CY, Schaber C, Crowley JR, Hsu FF, Carlson JR, et al. Malaria parasites produce volatile mosquito attractants. MBio. 2015;6(2).

18. Nyasembe VO, Teal PE, Mukabana WR, Tumlinson JH, Torto B. Behavioural response of the malaria vector Anopheles gambiae to host plant volatiles and synthetic blends. Parasit Vectors. 2012;5:234.

19. Bhavsar AP, Guttman JA, Finlay BB. Manipulation of host-cell pathways by bacterial pathogens. Nature. 2007:449:827-34.

20. Zhou J, Zeng L, Liu J, Xing D. Manipulation of the xanthophyll cycle increases plant susceptibility to Sclerotinia sclerotiorum. PLoS Pathog. 2015; 11(5):e1004878.

21. Groen SC, Jiang S, Murphy AM, Cunniffe NJ, Westwood JH, Davey MP, et al. Virus infection of plants alters pollinator preference: a payback for susceptible hosts? PLoS Pathog. 2016;12(8):e1005790. 
22. Kaushik M, Knowles SC, Webster JP. What makes a feline fatal in Toxoplasma gondii's fatal feline attraction? Infected rats choose wild cats. Integr Comp Biol. 2014;54(2):118-28.

23. Silverman JM, Clos J, Horakova E, Wang AY, Wiesgigl M, Kelly I, et al. Leishmania exosomes modulate innate and adaptive immune responses through effects on monocytes and dendritic cells. J Immunol. 2010; 185(9):5011-22

24. Hassani K, Olivier M. Immunomodulatory impact of Leishmania-induced macrophage exosomes: a comparative proteomic and functional analysis. PLoS Negl Trop Dis. 2013;7(5):e2185.

25. Gulati S, Ekland EH, Ruggles KV, Chan RB, Jayabalasingham B, Zhou B, et al. Profiling the essential nature of lipid metabolism in asexual blood and gametocyte stages of Plasmodium falciparum. Cell Host Microbe. 2015;18(3):371-81.

26. Malleret B, Li A, Zhang R, Tan KS, Suwanarusk R, Claser C, et al. Plasmodium vivax: restricted tropism and rapid remodeling of CD71-positive reticulocytes. Blood. 2015;125(8):1314-24.

27. Martin-Jaular L, Nakayasu ES, Ferrer M, Almeida IC, Del Portillo HA. Exosomes from Plasmodium yoelii-infected reticulocytes protect mice from lethal infections. PLoS One. 2011;6(10):e26588.

28. Regev-Rudzki N, Wilson DW, Carvalho TG, Sisquella X, Coleman BM, Rug M, et al. Cell-cell communication between malaria-infected red blood cells via exosome-like vesicles. Cell. 2013;153(5):1120-33.

29. Mantel P-Y, Hoang AN, Goldowitz I, Potashnikova D, Hamza B, Vorobjev I, et al. Malaria infected erythrocyte-derived microvesicles mediate cellular communication within the parasite population and with the host immune system. Cell Host Microbe. 2013;13(5):521-34.

30. Trager W, Jensen JB. Human malaria parasites in continuous culture. Science. 1976;193(4254):673-5.

31. Almanza A, Tayler N, Herrera L, Spadafora C. Automated synchronization of P. falciparum using a temperature cycling incubator. Curr Trends Biotechnol Pharm. 2011;5(2):1130-3.

32. Garcia-Silva MR, Neves RF C d, Cabrera-Cabrera F, Sanguinetti J, Medeiros LC, Robello C, et al. Extracellular vesicles shed by Trypanosoma cruzi are linked to small RNA pathways, life cycle regulation, and susceptibility to infection of mammalian cells. Parasitol Res. 2014;113(1):285-304.

33. Orozco AF, Lewis DE. Flow cytometric analysis of circulating microparticles in plasma. Cytometry A. 2010;77(6):502-14.

34. Gallart-Palau X, Serra A, Wong AS, Sandin S, Lai MK, Chen CP, et al. Extracellular vesicles are rapidly purified from human plasma by protein organic solvent precipitation (PROSPR). Sci Rep. 2015:5:14664.

35. Lenassi M, Cagney G, Liao M, Vaupotic T, Bartholomeeusen K, Cheng Y, et al. HIV Nef is secreted in exosomes and triggers apoptosis in bystander CD4 + T cells. Traffic. 2010;11(1):110-22.

36. Blackman MJ, Heidrich HG, Donachie S, McBride JS, Holder AA. A single fragment of a malaria merozoite surface protein remains on the parasite during red cell invasion and is the target of invasion-inhibiting antibodies. J Exp Med. 1990;172(1):379-82

37. Hernandez-Hernandez A, Rodriguez MC, Lopez-Revuelta A, Sanchez-Gallego $\mathrm{Jl}$, Shnyrov V, Llanillo $\mathrm{M}$, et al. Alterations in erythrocyte membrane protein composition in advanced non-small cell lung cancer. Blood Cells Mol Dis. 2006;36(3):355-63.

38. Kim H, Cho W-J, Ahn J-S, Cho D-H, Cha Y-J. Identification of radiolytic marker compounds in the irradiated beef extract powder by volatile analysis. Microchem J. 2005;80(2):127-37.

39. Schorey JS, Cheng Y, Singh PP, Smith VL. Exosomes and other extracellular vesicles in host-pathogen interactions. EMBO Rep. 2015;16(1):24-43.

40. Schaffler I, Steiner KE, Haid M, van Berkel SS, Gerlach G, Johnson SD, et al. Diacetin, a reliable cue and private communication channel in a specialized pollination system. Sci Rep. 2015;5:12779.

41. Mochalski P, King J, Klieber M, Unterkofler K, Hinterhuber H, Baumann M, et al. Blood and breath levels of selected volatile organic compounds in healthy volunteers. Analyst. 2013;138(7):2134-45.

42. Nyasembe VO, Tchouassi DP, Kirwa HK, Foster WA, Teal PE, Borgemeister C, et al. Development and assessment of plant-based synthetic odor baits for surveillance and control of malaria vectors. PLoS One. 2014;9(2):e89818.

43. Mochalski $P$, King J, Haas M, Unterkofler K, Amann A, Mayer G. Blood and breath profiles of volatile organic compounds in patients with end-stage renal disease. BMC Nephrol. 2014;15:43.

44. Mochalski P, King J, Unterkofler K, Hinterhuber H, Amann A. Emission rates of selected volatile organic compounds from skin of healthy volunteers. J Chromatogr B Analyt Technol Biomed Life Sci. 2014;959:62-70.
45. Vautz W, Slodzynski R, Hariharan C, Seifert L, Nolte J, Fobbe R, et al Detection of metabolites of trapped humans using ion mobility spectrometry coupled with gas chromatography. Anal Chem. 2013;85(4):2135-42

46. Li N, Deng C, Yin X, Yao N, Shen X, Zhang X. Gas chromatography-mass spectrometric analysis of hexanal and heptanal in human blood by headspace single-drop microextraction with droplet derivatization. Anal Biochem. 2005;342(2):318-26.

47. Deng C, Zhang X, Li N. Investigation of volatile biomarkers in lung cancer blood using solid-phase microextraction and capillary gas chromatographymass spectrometry. J Chromatogr B Analyt Technol Biomed Life Sci. 2004; 808(2):269-77

48. Fuchs $\mathrm{P}$, Loeseken C, Schubert JK, Miekisch W. Breath gas aldehydes as biomarkers of lung cancer. Int J Cancer. 2010;126(11):2663-70.

49. Berna AZ, McCarthy JS, Wang RX, Saliba KJ, Bravo FG, Cassells J, et al. Analysis of breath specimens for biomarkers of Plasmodium falciparum infection. J Infect Dis. 2015;212(7):1120-8.

50. Buffinton GD, Hunt NH, Cowden WB, Clark IA. Detection of short-chain carbonyl products of lipid peroxidation from malaria-parasite (Plasmodium vinckei)-infected red blood cells exposed to oxidative stress. Biochem J. 1988;249(1):63-8.

51. Keller JN, Pang Z, Geddes JW, Begley JG, Germeyer A, Waeg G, et al. Impairment of glucose and glutamate transport and induction of mitochondrial oxidative stress and dysfunction in synaptosomes by amyloid $\beta$-Peptide: Role of the lipid peroxidation product 4-hydroxynonenal. J Neurochem. 1997:69(1):273-84

52. Frankel EN, Tappel AL. Headspace gas chromatography of volatile lipid peroxidation products from human red blood cell membranes. Lipids. 1991;26(6):479-84

\section{Submit your next manuscript to BioMed Central and we will help you at every step:}

- We accept pre-submission inquiries

- Our selector tool helps you to find the most relevant journal

- We provide round the clock customer support

- Convenient online submission

- Thorough peer review

- Inclusion in PubMed and all major indexing services

- Maximum visibility for your research

Submit your manuscript at www.biomedcentral.com/submit

) Biomed Central 